\title{
Modeling the Mechanical Properties of Functionalized Carbon Nanotubes and Their Composites: Design at the Atomic Level
}

\author{
Qing-Sheng Yang, ${ }^{1}$ Bing-Qi Li, ${ }^{2}$ Xiao-Qiao He, ${ }^{3}$ and Yiu-Wing Mai ${ }^{4}$ \\ ${ }^{1}$ Department of Engineering Mechanics, Beijing University of Technology, Beijing 100124, China \\ ${ }^{2}$ China Institute of Water Resources and Hydropower Research, Beijing 100038, China \\ ${ }^{3}$ Department of Building and Construction, City University of Hong Kong, Tat Chee Avenue, Kowloon, Hong Kong \\ ${ }^{4}$ Centre for Advanced Materials Technology (CAMT), School of Aerospace, Mechanical and Mechatronic Engineering, University of \\ Sydney, Sydney, NSW 2006, Australia \\ Correspondence should be addressed to Xiao-Qiao He; bcxqhe@cityu.edu.hk
}

Received 31 October 2013; Accepted 13 January 2014; Published 26 February 2014

Academic Editor: Hanxing Zhu

Copyright (C) 2014 Qing-Sheng Yang et al. This is an open access article distributed under the Creative Commons Attribution License, which permits unrestricted use, distribution, and reproduction in any medium, provided the original work is properly cited.

\begin{abstract}
This investigation focuses on the design of functionalization configuration at the atomic level to determine the influence of atomic structure on the mechanical properties of functionalized carbon nanotubes (F-CNTs) and their composites. Tension and compressive buckling behaviors of different configurations of CNTs functionalized by $\mathrm{H}$ atoms are studied by a molecular dynamics (MD) method. It is shown that $\mathrm{H}$-atom functionalization reduces Young's modulus of CNTs, but Young's modulus is not sensitive to the functionalization configuration. The configuration does, however, affect the tensile strength and critical buckling stress of CNTs. Further, the stress-strain relations of composites reinforced by nonfunctionalized and various functionalized CNTs are analyzed.
\end{abstract}

\section{Introduction}

Carbon nanotubes (CNTs), discovered in the early 1990s, have promising mechanical, thermal, and electrical properties and thus have many applications in engineering $[1$, 2]. The properties of CNTs can be simulated by molecular dynamics (MD) [3], molecular mechanics (MM) [4], and other approximation methods $[5,6]$.

A primary application of CNTs is in the fabrication of composites, in which the CNTs are embedded in a polymer matrix. However, it has been shown that the interfacial adhesion of the polymer and the CNT is weak, which results in a lower efficiency in transferring the external load to the CNT [7]. Chemical functionalization of the CNTs is an efficient way to achieve stronger interface adhesion between CNTs and the surrounding polymer chains in such composites. Xie et al. [8] and Daniel et al. [9] reviewed some of the functionalizing methods available. Chemical functionalization methods allow specific carbon atoms to be selected for the sidewall modification of CNTs by hybridizing the functional group or a particular atom, such as the hydrogen atom.

Some research also indicates that functionalization of CNTs can lead to degradation of some of their properties, depending on the chirality and functionalization configuration. Work by Garg and Sinnott [10] shows that the functionalization of CNTs decreases the maximum buckling load by about $15 \%$ despite the tubule helical structures and regardless of the radius. Buffa et al. [11] state that surface defects are induced by functionalization, which leads to loss of tensile strength in the CNTs. The molecular dynamics simulation of CNTs by Xin et al. [12] indicates that the buckling behavior of SWCNTs is affected by vacancy defects owing to chemical functionalization. Molecular mechanics investigations by Kuang et al. [13, 14] and He et al. [15] reveal a reduction in Young's modulus of up to $37 \%$ after functionalization when the functional group is densely distributed. Functionalization may also weaken the fracture strain and tensile strength [16] and the torsion behavior of CNTs [17]. 
Although existing investigations on functionalized CNTs focus on the mechanical property reduction of CNTs, several problems remain unsolved. In particular, the desired functionalization configuration that results in optimum mechanical properties of CNTs is still unknown. Indeed, for design at the atomic level of CNTs, it is very important to understand the variation of mechanical properties with the functionalization configuration of CNTs. In this paper, the overall performance, including tensile and buckling behaviors, of CNTs with different functionalization configuration and their composites are investigated by MD simulations. Optimum configuration of CNT functionalization with hydrogen atoms is explored by varying the atomic design of functionalized CNTs. Then the average properties of functionalized CNT/polyethylene composite are studied to understand the effect of atomic structure on the overall properties of the composite.

\section{MD Simulation Method}

The main steps in molecular dynamics simulation include the selection of the potential function, setting of initial conditions, and balance of calculations [18].

In MD simulation, the movement of atoms at the nanoscale can be described by the classical Newton equation of motion:

$$
m_{i} \frac{\partial^{2} r_{i}}{\partial t^{2}}=F_{i}
$$

where $m_{i}$ is the mass of an atom, $r_{i}$ coordinate of an atom, and $F_{i}$ combination of forces acting on atom $i$ from the surrounding atoms. In molecular dynamics,

$$
F_{i}=-\frac{\partial V}{\partial r_{i}}
$$

where $V$ is the potential function between the atoms.

The research object of molecular dynamics is always a multiparticle system. The number of particles in a system that can be simulated is finite due to the influence of the external conditions. However, the rules of statistical physics are still tenable, and thus suitable ensemble must be selected for the simulation.

The main ensembles used in MD simulation are Microcanonical Ensemble (NVE), Canonical Ensemble (NVT), Isobaric-isothermal Ensemble (NPT), and Pressure-enthalpy Ensemble (NPH) [18]. NVT is adopted for the simulation conducted in this work.

With the NVT, the particle number, volume, temperature, and total momentum are all conserved. However, the total energy of a system is not conserved at a constant temperature: energy exchange must occur between the system and the external environment to maintain a constant temperature. The method adopted here achieves a heat balance between the system and the external heat bath using a variable that describes the interaction between the system and the external environment [19]. Thus (1) becomes

$$
\ddot{r}=\frac{F_{i}}{m}-\eta \dot{r}_{i},
$$

where $\dot{\eta}=\left(1 / \tau^{2}\right)\left(T / T_{C}-1\right)$ and $\tau=\sqrt{Q / 3 N k_{B} T_{C}} \cdot \tau$ is the relaxation time of the heat bath that determines the rate at which the system tends to a constant temperature and that can be evaluated once or several times in time-steps. $k_{B}=$ $1.3806 \times 10^{-23} \mathrm{~J} / \mathrm{K}$ is the Boltzmann constant.

Because the model is made of CNTs and functionalized atoms, only $\mathrm{C}$ and $\mathrm{H}$ atoms are present. The AIREBO potential for the $\mathrm{C}$ and $\mathrm{H}$ atoms is thus adopted. This potential, which includes the Lennard-Jones pair potential, is a modified REBO potential.

The AIREBO potential [18] can be written as

$$
E=\frac{1}{2} \sum_{i} \sum_{j \neq i}\left[E_{i j}^{\mathrm{REB}}+E_{i j}^{L J}+\sum_{k \neq i, j l} \sum_{\neq i, j, k} E_{k i j l}^{\mathrm{TOR}}\right],
$$

where $E_{i j}^{\mathrm{REB}}$ gives the model its reactive capabilities and only describes short-range $\mathrm{C}-\mathrm{C}, \mathrm{C}-\mathrm{H}$, and $\mathrm{H}-\mathrm{H}$ interactions $(r<$ $2 \AA)$, and $E_{i j}^{L J}$ adds longer range interactions $\left(2 \AA<r<r_{\text {cut }}\right)$ using a form similar to the standard Lennard-Jones potential. $E_{k i j l}^{\mathrm{TOR}}$ is an explicit four-body potential that describes the various dihedral angle preferences in the hydrocarbon configurations. The Lennard-Jones potential is

$$
E=4 \varepsilon\left[\left(\frac{\sigma}{r}\right)^{12}-\left(\frac{\sigma}{r}\right)^{6}\right], \quad r<r_{\text {cut }} .
$$

Different parameters are given for $\varepsilon$ and $\sigma$ based on the atoms that are included in the foregoing formula.

Finite difference algorithms can be applied to determine the movement of the atoms. Some commonly used algorithms include the Verlet algorithm, Beeman algorithm, and Leapfrog algorithm $[18,19]$. The most extensively used Verlet algorithm is employed here.

Suppose that the position of an atom in a molecular system is $r(t)$ at time $t$. The position of the atom can then be obtained by Taylor expansion for time $(t+\delta t)$ as

$$
r(t+\delta t)=r_{i}(t)+\delta t v_{i}(t)+\frac{1}{2} \delta t^{2} a_{i}(t)+\cdots
$$

and for time $(t-\delta t)$ as

$$
r(t-\delta t)=r_{i}(t)-\delta t v_{i}(t)+\frac{1}{2} \delta t^{2} a_{i}(t)-\cdots
$$

By adding (6) and (7), we can obtain the position of the atom at $(t+\delta t)$ as

$$
r(t+\delta t)=2 r_{i}(t)-r_{i}(t-\delta t)+\delta t^{2} \frac{F_{i}(t)}{m_{i}} .
$$

The velocity of the atom can be calculated from

$$
v(t)=\frac{[r(t+\delta t)-r(t-\delta t)]}{2 \delta t} .
$$

\section{Model of Functionalized CNTs}

CNTs have periodicity in the axial direction. The configuration of a CNT is characterized by $(m, n)$, which governs 


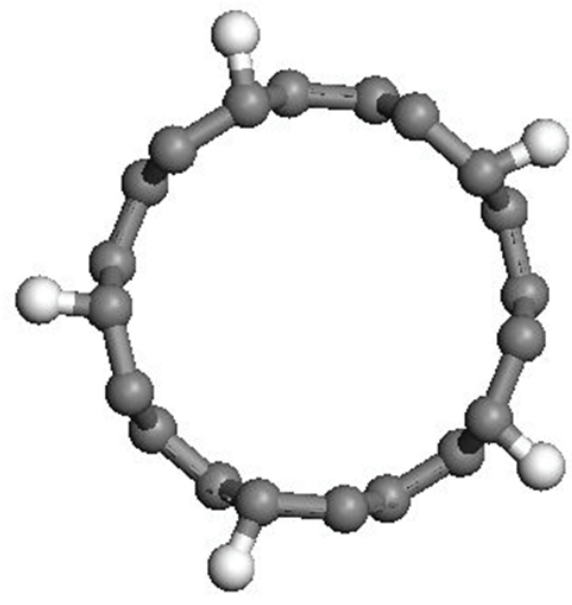

Figure 1: Cross-section of CNT functionalized by $\mathrm{H}$ atoms.

the diameter, helix angle, lattice constant, and physical properties. In the present study, for simplicity, a functionalized $(5,5) \mathrm{CNT}$ is examined to characterize the tension and buckling behavior by using molecular dynamics simulation. The radius and length of the CNT are $0.339 \mathrm{~nm}$ and $5.903 \mathrm{~nm}$, respectively. The cross-section of CNT functionalized by the $\mathrm{H}$ atoms is shown in Figure 1 where 5 atoms of 20 carbon atoms in a ring are functionalized by $\mathrm{H}$ atoms. The functionalized length is half the total length of the CNT. Thus the functionalized density is $12.5 \%$. Different functionalization configurations are adopted which have the same atomicity of $\mathrm{H}$ atoms. The $\mathrm{H}$ atoms in the model are divided into one, two, three, and six separate segments along the CNT axial direction, which are labeled F-CNT-1, F-CNT-2, F-CNT-3, and F-CNT-4, as shown in Figure 2.

In the simulation, the CNT and the $\mathrm{H}$ atoms are modeled using the AIREBO potential. The NVT ensemble is employed to keep the volume and temperature constant, and the Nose-Hoover method [20] is used to control the temperature at absolute zero, which eliminates the influence of thermal activation. The Verlet algorithm is adopted to solve the molecular dynamics equations in the simulation procedure. The same boundary condition is imposed on the CNTs for all the functionalization configurations: the two layers of atoms at the bottom are fixed and displacements are imposed on the atoms in the two layers at the top of the model. Young's modulus, buckling configurations, and critical buckling strain are obtained. The force applied on the atoms can also be calculated by this MD simulation. Then a resultant force of CNT can be obtained by adding all forces on the atoms along certain direction. The stress of CNT is defined as the resultant force subjected by all atoms divided by the volume of CNT.

\section{Properties of Functionalized CNTs}

The tensile behavior of a virgin CNT is determined first to verify the reliability of the present MD simulation. Young's modulus obtained is $1.03 \mathrm{TPa}$ in agreement with the values

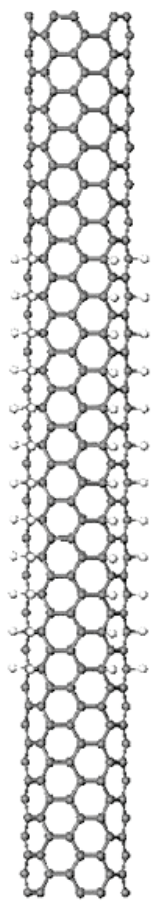

F-CNT-1

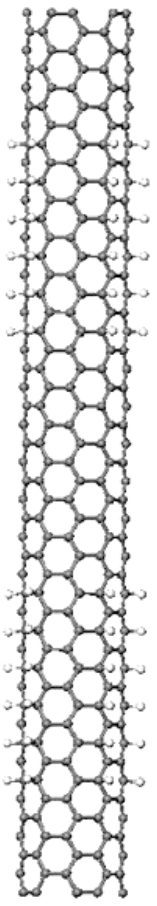

F-CNT-2

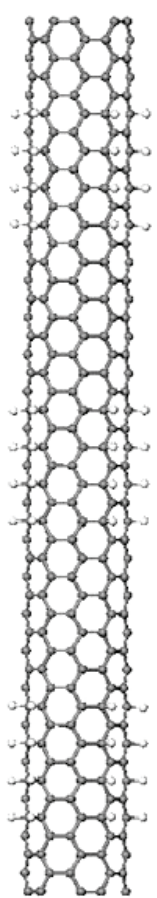

F-CNT-3

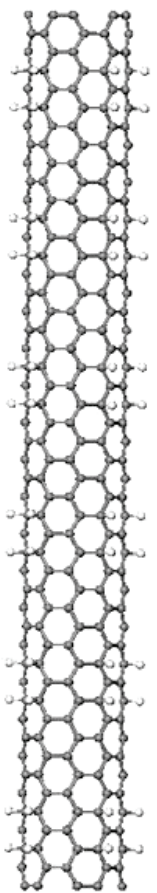

F-CNT-4
Figure 2: Functionalized CNT models.

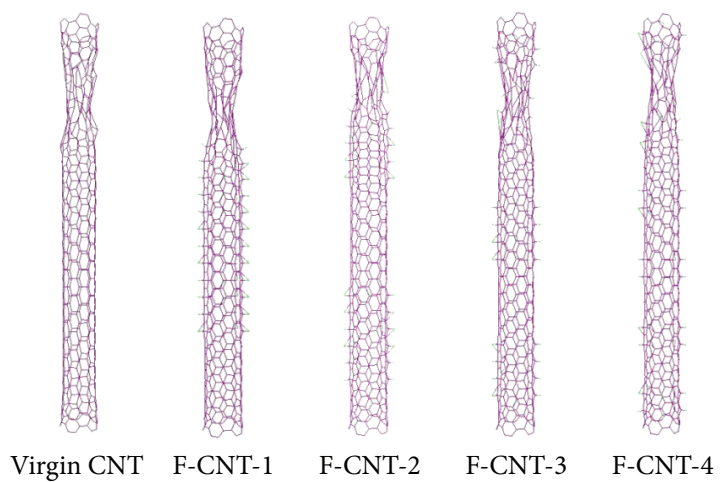

FIGURE 3: Tensile deformation of virgin CNT and functionalized CNTs.

reported in $[5,14-16]$. It can be seen from the simulation that when the models are extended, the hexagons begin to deform. Necking then occurs, after which, the models fracture completely. Figure 3 shows the deformation of the five models. In model F-CNT-1, some top functionalized atoms undergo necking. In model F-CNT-2, the functionalized atoms are divided into two parts, and necking occurs at the top of the upper segment. In models F-CNT-3 and F-CNT-4, necking happens at the bottom of the upper functionalized segment. Comparisons of the deformation of the five models show that the transition region between the functionalized and nonfunctionalized atoms is weak leading to necking and fracture easily. 
TABLE 1: Tensile properties of F-CNTs with different functionalized configurations.

\begin{tabular}{|c|c|c|c|c|c|}
\hline & CNT & F-CNT-1 & F-CNT-2 & F-CNT-3 & F-CNT-4 \\
\hline Young's modulus (TPa) & 1.03 & 0.68 & 0.692 & 0.669 & 0.676 \\
\hline Failure strain & 0.264 & 0.266 & 0.25 & 0.26 & 0.239 \\
\hline Maximum stress $(\mathrm{GPa})$ & 188 & 134 & 117 & 123 & 112 \\
\hline Maximum potential energy increment $(\mathrm{eV})$ & 597.59 & 593.53 & 554.25 & 582.91 & 504.35 \\
\hline
\end{tabular}

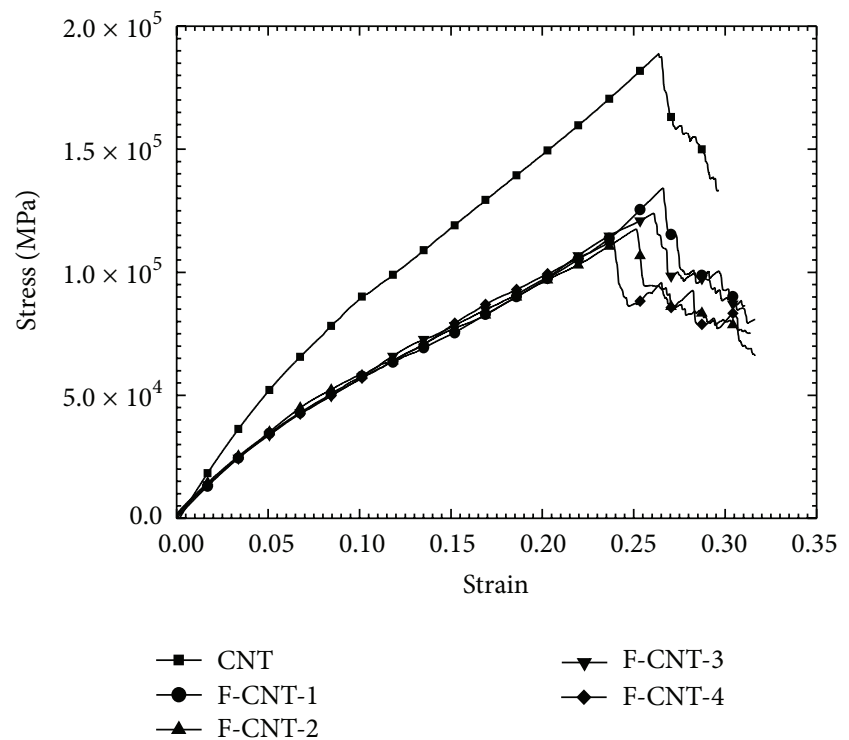

FIGURE 4: Variation of tensile stress versus strain of CNTs with different functionalization configurations.

Figure 4 shows the variations of stress with strain of the CNTs with different functionalization configurations. The stress increases with increasing strain. The range of stress increase in the nonfunctionalized CNTs with strain is larger than those of the functionalized CNTs. Thus, at the same strain, the stress in F-CNT-1 is larger than all of other functionalized configurations. Also, F-CNT-4 sustains the lowest stress and fractures first.

Young's modulus of CNTs with different functionalization configuration can be obtained from Figure 4 and shown in Table 1. Functionalization decreases markedly Young's modulus and tensile strength of the CNTs, but the functionalization configurations with the same intensity do not have any significant effect. Young's modulus of the functionalized CNTs is almost always degraded by $\sim 33 \%$ in these models. These results are consistent with prior studies [14, 21], which depend on the functionalization degree, hybridized atom, and chirality of CNT. The failure strains and maximum stresses are listed in Table 1 for the extension of virgin and functionalized CNTs. It is shown that the theoretical strength of CNT decreases by $28-40 \%$ due to the functionalization, while the variations of the failure strains are indistinguishable.

Buckling of the CNTs under compression with different functionalization configurations is then simulated using the same method. In the simulation, multiple necking occurs until the models finally bend, as shown in Figure 5. In

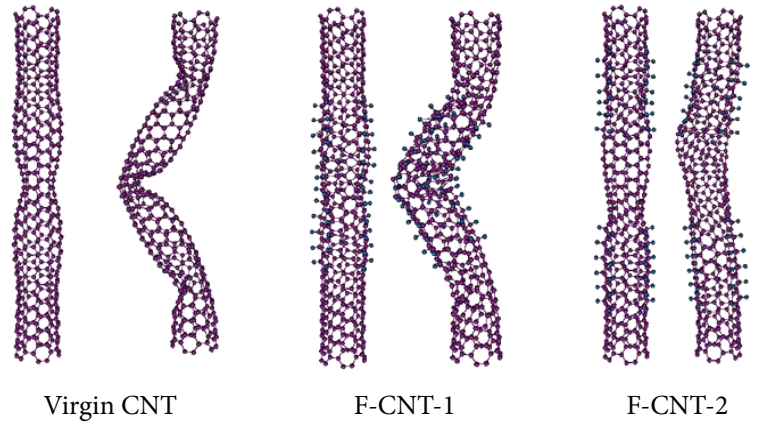

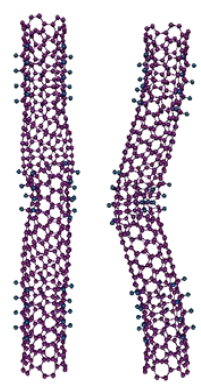

F-CNT-3

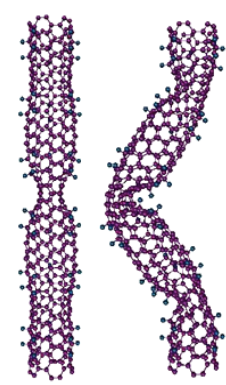

F-CNT-4
FIGURE 5: Buckling deformation of CNTs with different functionalization configurations.

F-CNT-1, necking is located in the middle, and bending occurs at the boundary of the functionalized atoms. In FCNT-2, necking occurs at the top of the lower functionalized segment, and bending appears at the bottom of the upper functionalized segment. In F-CNT-3, the bend is located in the middle, as in F-CNT-1. Buckling of F-CNT-4 is similar to that of the non-functionalized CNT. This comparison shows that the transition region between the non-functionalized and functionalized atoms is weak and breaks easily.

The strain of the models before necking occurs is called the critical buckling strain. Table 2 shows the critical buckling strain, maximum stress (theoretical strength), and maximum potential energy increment of CNTs for different functionalization configurations. There is little difference (often less than $10 \%$ ) between the strain of the functionalized and nonfunctionalized models. But functionalization can reduce the maximum stress of the CNT by $45-52 \%$.

Figure 6 shows the curves of potential energy increment and strain. When the potential energy increment drops, necking occurs, and the corresponding strain is the critical buckling strain. It can be seen from these curves that the potential energy increment also increases with strain increment after the critical buckling strain; but this change is 
TABLE 2: Mechanical properties of F-CNTs with different functionalized configurations.

\begin{tabular}{|c|c|c|c|c|c|}
\hline & CNT & F-CNT-1 & F-CNT-2 & F-CNT-3 & F-CNT-4 \\
\hline Critical buckling strain & 0.058 & 0.062 & 0.062 & 0.061 & 0.053 \\
\hline Maximum stress $(\mathrm{GPa})$ & 125 & 68 & 67 & 67 & 60 \\
\hline Maximum potential energy increment $(\mathrm{eV})$ & 73.70 & 72.96 & 70.00 & 72.50 & 57.74 \\
\hline
\end{tabular}

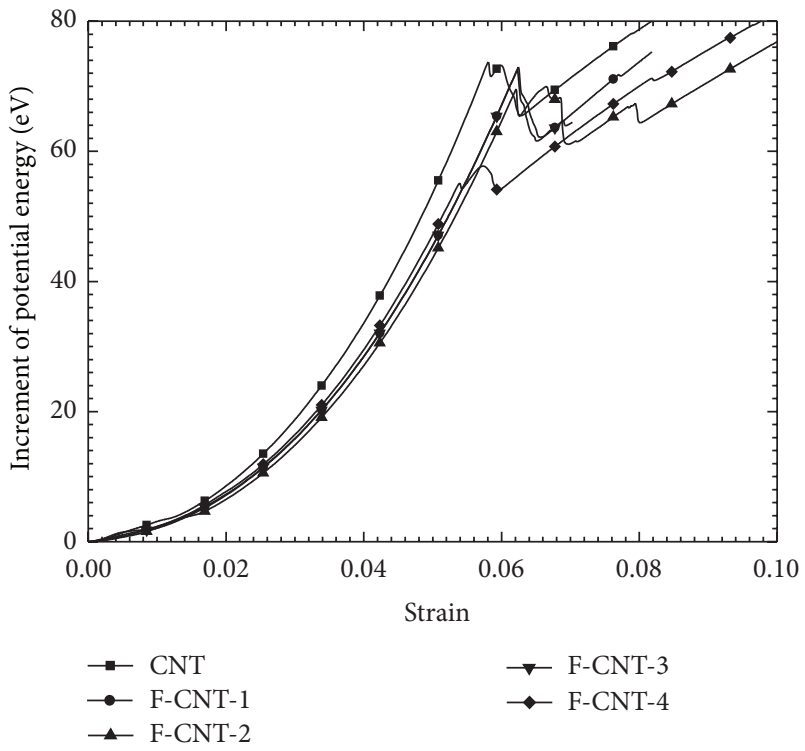

FIGURE 6: Variation of potential energy increment versus strain of CNTs with different functionalized configurations.

smaller because the initial buckling releases a large amount of energy, so that the energy release in subsequent buckling gradually decreases. The buckling strain of F-CNT-1 is larger than other models with F-CNT-4 being the smallest (see Figure 6). Under compression, the maximum buckling stress of the nonfunctionalized CNT is the largest, followed by FCNT-1 as the second largest and F-CNT-4 as the smallest (see Figure 7).

It must be noted that in the above simulation studies on the tensile and compressive behaviors, the $\mathrm{H}$-atoms represent the functionalization. In the present model, CNTs with these $\mathrm{H}$-atoms are considered for "stress-free" boundary conditions. Though the functionalization leads to decreases of the modulus and strength of CNTs, the interfacial adhesion between $\mathrm{CNT}$ and polymer matrix may improve owing to the functionalized CNTs. The property evaluation of functionalized CNTs is significant to the determination of in situ properties of the components prior to the prediction of effective properties of CNT composites.

\section{Simulation of CNT/Polyethylene Composite}

A composite model can be built by embedding the nonfunctionalized and functionalized CNTs into the polyethylene (PE) matrix. Figure 8 shows a molecular dynamics model of the CNT/PE composite where there are four-layer (total 150) molecules of PE surrounding the CNT. The distances between

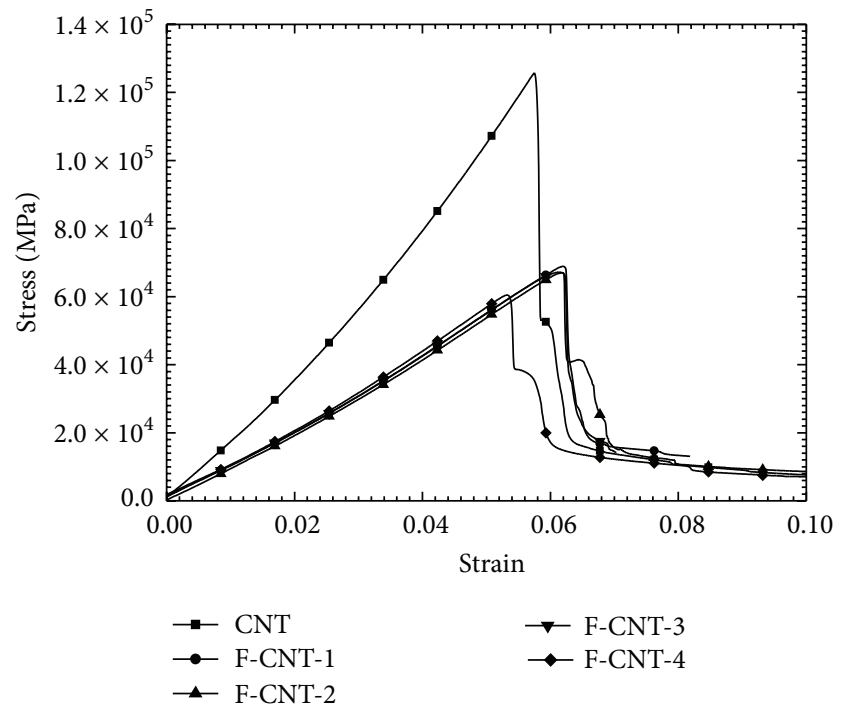

FIGURE 7: Variation of compression stress versus strain of CNTs with different functionalization configurations.

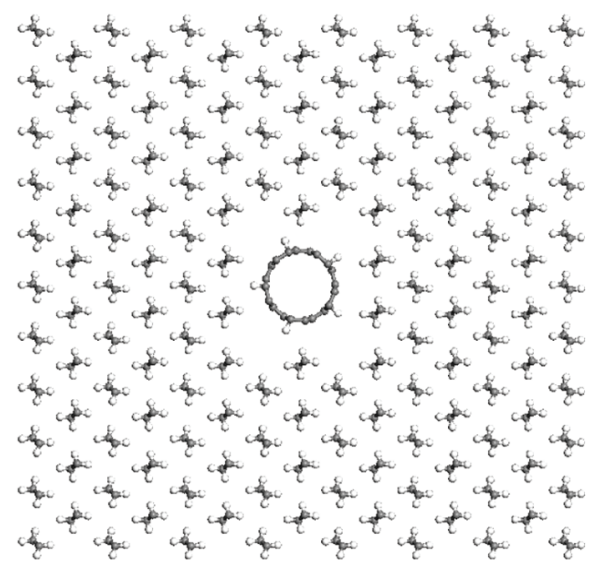

FIGURE 8: CNT/polyethylene composite.

the carbon atoms of CNT and PE are $0.48 \mathrm{~nm}$ in the $x$ direction and $0.36 \mathrm{~nm}$ in the $y$-direction, respectively. Several studies were performed on the interfacial adhesion behavior of functionalized-CNT/PE composites $[21,22]$. In the present work, the overall properties of the composite are emphasized so as to consider the influence of functionalized CNTs on the overall properties of the CNT/PE composites.

After free relaxation of the CNT/PE composite corresponding to a stable state with minimum energy, uniform tensile displacement is applied as shown in Figure 9. This 


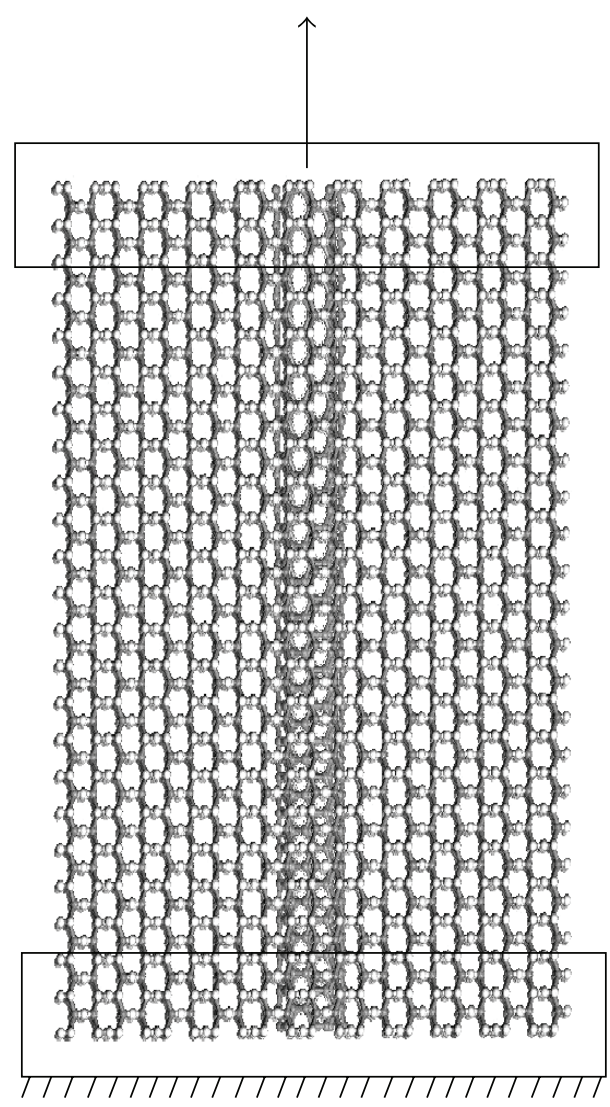

FIGURE 9: Boundary condition of composite.

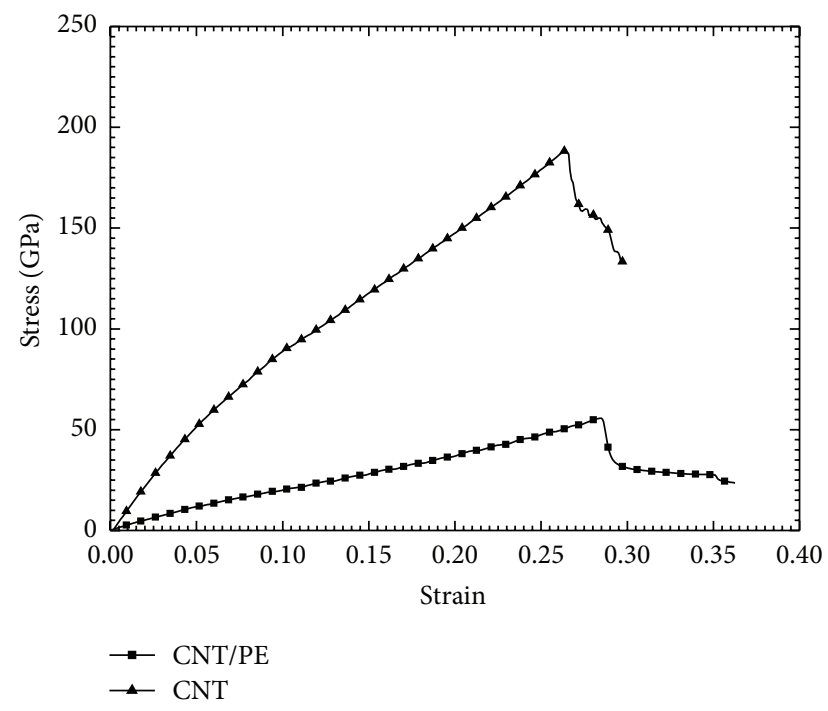

FIGURE 10: Tensile stress-strain curves of CNT/PE composite.

displacement boundary condition will induce a uniform tension in the CNT and PE. The uniaxial stress-strain curve of the composite can be obtained by $\mathrm{MD}$ simulation.

Comparisons of the stress-strain curves of the CNT and the composite reinforced by virgin or nonfunctionalized CNTs are given in Figure 10. The volume fraction of CNT

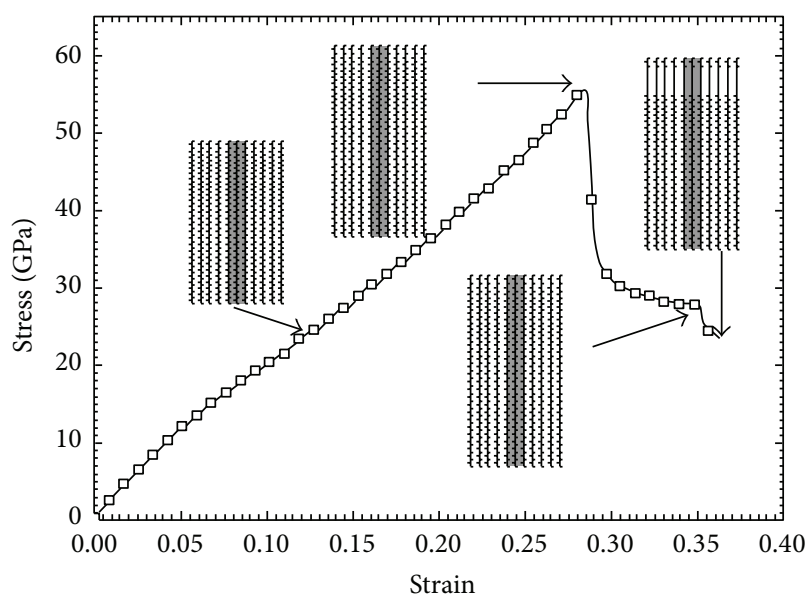

FIgURE 11: Tensile deformation and failure of CNT/PE composite.

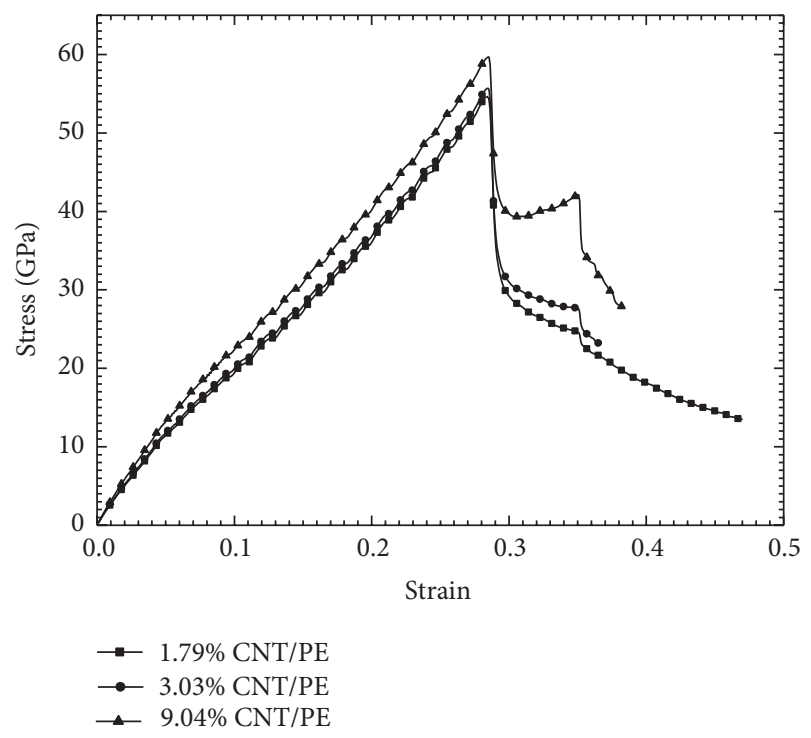

FIGURE 12: Variation of tensile stress versus strain of CNT/PE composites with different volume fractions.

is $9.04 \%$ and the CNT is considered as a solid cylinder. The calculated Young's modulus of the composite is $\sim 244 \mathrm{MPa}$. This result does not agree with the rule of mixtures for Young's modulus. However, the composite failure strain is nearly equal to that of CNT under tension. This is in agreement with the conclusion of the molecular mechanics method [23].

Figure 11 gives the deformed profiles of the CNT/PE composite at different deformation and failure stages. It can be seen that the CNT dominates the deformation of the composite as PE has very low modulus and high ductility. The peak stress borne by the composite can be obtained as the CNT is broken. Then the stress rapidly decreases until complete failure of the composite. The strength of the composite is naturally affected by the volume fraction of the CNTs (see Figure 12). Three volume fractions of CNTs, that is, 1.79, 3.03, and $9.04 \%$, are considered. It can be seen that the stress-strain 


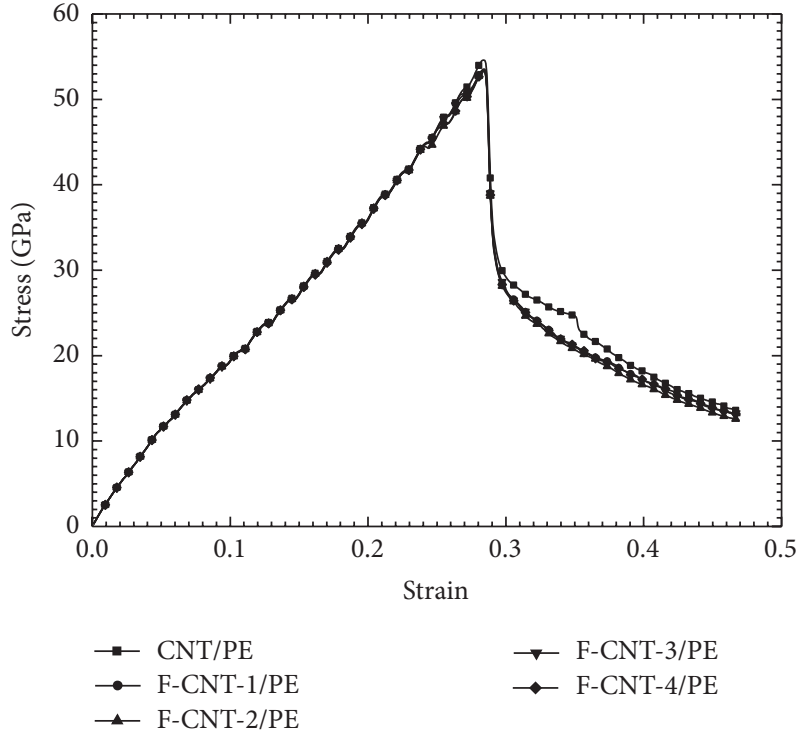

FIGURE 13: Variation of tensile stress versus strain of CNT/PE composites with different functionalization configurations.

curve is the highest for the composite with the highest level of CNTs, thus indicating the largest composite modulus and strength.

Figure 13 displays the stress-strain relations of CNT/PE composites for different functionalized configurations. Clearly, Young's moduli of the composites reinforced by nonfunctionalized and various functionalized CNTs are very similar though Young's modulus of CNT is reduced by $\mathrm{H}$-atom functionalization. This means that the $\mathrm{H}$-atom functionalization hardly affects the overall elastic properties of continuous CNT/PE composites under uniform axial deformation. Also, the functionalization configurations, which introduce different interfacial adhesion between CNT and PE, do not influence the overall stress-strain behavior of the composites, since there are no large differences in the stress transfer capacity at the interface.

The compression and buckling behaviors of CNT/PE and F-CNT/PE composites are studied. With different volume fractions of CNT, the first buckling of CNT/PE corresponds to the yield of $\mathrm{PE}$ as shown in Figure 14. The second buckling at $6 \%$ compression strain relates to the failure of the CNT, which leads to critical buckling of the whole composite.

Figure 15 illustrates the relations of compression stress and strain of CNT/PE composites with different functionalization configuration, which reduces the load-bearing capacity just before and immediately after the peak stress.

\section{Concluding Remarks}

The tension and compression behaviors of CNTs with different functionalized configurations are simulated with a molecular dynamics method. The stress-strain relations of the F-CNT/PE and CNT/PE composites are obtained. The main findings are given below.

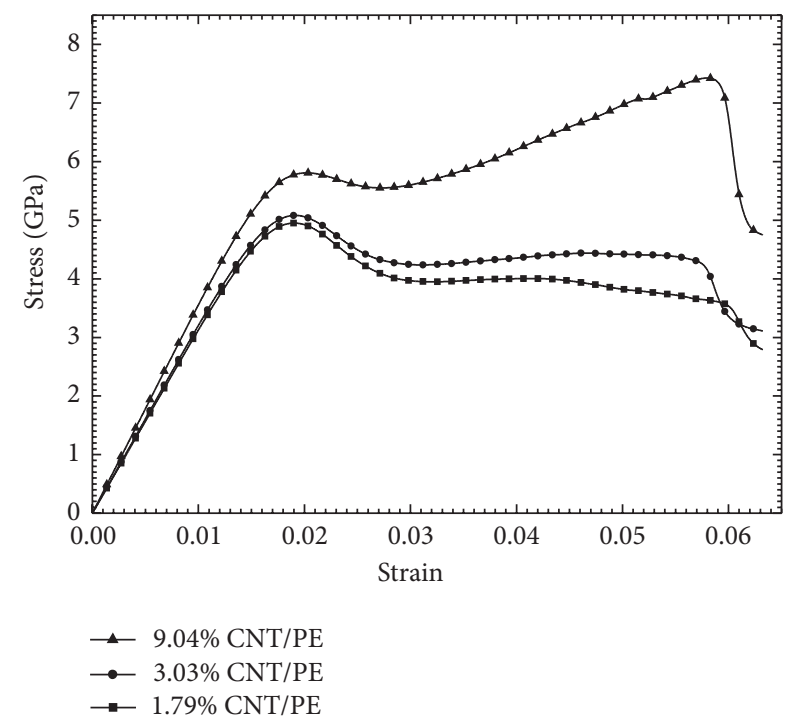

FIGURE 14: Variation of compression stress versus strain of CNT/PE composites with different volume fractions of CNTs.

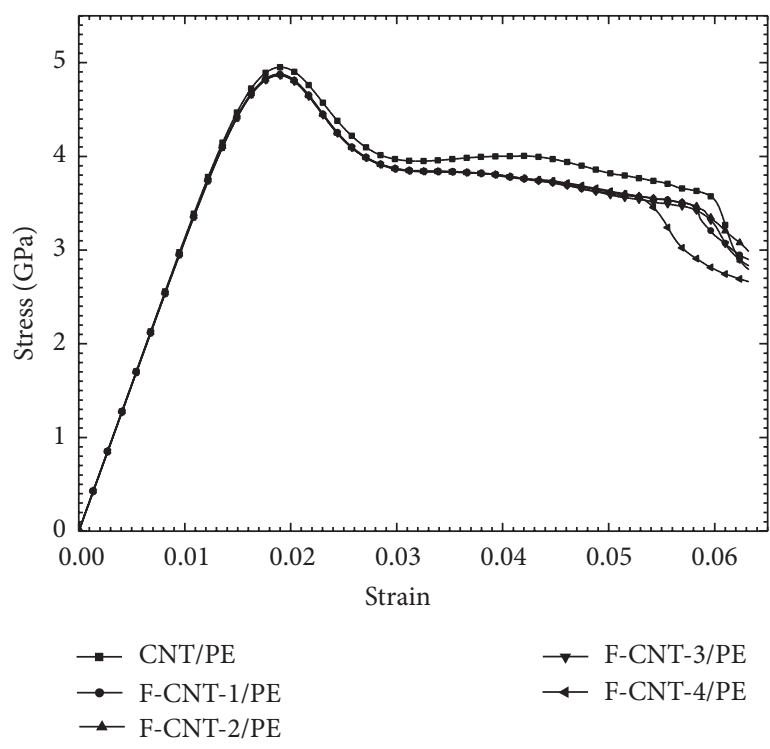

FIGURE 15: Variation of compression stress versus strain of CNT/PE composites with different functionalization configurations.

(a) The functionalization of CNTs decreases their Young's modulus by $33 \%$ at a functionalized density of $12.5 \%$. However, functionalization configurations have no effect on the modulus.

(b) Tensile strength and fracture strain of F-CNTs differ across functionalization configurations. The maximum stress of a functionalized CNT is smaller than that of a nonfunctionalized CNT. In terms of functionalization configurations, the more dispersed functionalized atoms yield lower strength and failure strain of CNTs. 
(c) Functionalization configuration has no obvious effect on the critical buckling strai, but does affect the buckling mode of CNTs. The transition region between functionalized and nonfunctionalized atoms is weaker than other regions of the CNTs. Thus, functionalization yields lower maximum buckling stress.

(d) Unlike CNT/PE composites, functionalization of CNTs hardly affects Young's modulus and maximum stress of F-CNT/PE composites. Also, the failure strain of the composites is nearly equal to that of the CNTs. There is almost no effect of functionalization configurations of CNTs on the overall properties of the composites under both tension and compression.

\section{Conflict of Interests}

The authors declare that there is no conflict of interests regarding the publication of this paper.

\section{Acknowledgments}

Financial support from the Natural Science Foundation of China under Grant no. 11172012, 11372264 and the Specialized Research Fund for the Doctoral Program of Higher Education of China under Grant no. 20101103110005 is gratefully acknowledged.

\section{References}

[1] R. H. Baughman, A. A. Zakhidov, and W. A. de Heer, "Carbon nanotubes - the route toward applications," Science, vol. 297, no. 5582, pp. 787-792, 2002.

[2] C. Li, E. T. Thostenson, and T.-W. Chou, "Sensors and actuators based on carbon nanotubes and their composites: a review," Composites Science and Technology, vol. 68, no. 6, pp. 1227-1249, 2008.

[3] B. I. Yakobson, C. J. Brabec, and J. Bernbolc, "Nanomechanics of carbon tubes: instabilities beyond linear response," Physical Review Letters, vol. 76, no. 14, pp. 2511-2514, 1996.

[4] H. Sun, "Compass: an ab initio force-field optimized for condensed-phase applications-overview with details on alkane and benzene compounds," Journal of Physical Chemistry B, vol. 102, no. 38, pp. 7338-7364, 1998.

[5] C. Li and T.-W. Chou, "A structural mechanics approach for the analysis of carbon nanotubes," International Journal of Solids and Structures, vol. 40, no. 10, pp. 2487-2499, 2003.

[6] G. I. Giannopoulos, P. A. Kakavas, and N. K. Anifantis, "Evaluation of the effective mechanical properties of single walled carbon nanotubes using a spring based finite element approach," Computational Materials Science, vol. 41, no. 4, pp. 561-569, 2008.

[7] S. J. V. Frankland and V. M. Harik, "Analysis of carbon nanotube pull-out from a polymer matrix," Surface Science, vol. 525, no. 13, pp. L103-L108, 2003.

[8] X.-L. Xie, Y.-W. Mai, and X.-P. Zhou, "Dispersion and alignment of carbon nanotubes in polymer matrix: a review," Materials Science and Engineering R, vol. 49, no. 4, pp. 89-112, 2005.

[9] S. Daniel, T. P. Rao, K. S. Rao et al., "A review of DNA functionalized/grafted carbon nanotubes and their characterization," Sensors and Actuators B, vol. 122, no. 2, pp. 672-682, 2007.
[10] A. Garg and S. B. Sinnott, "Effect of chemical functionalization on the mechanical properties of carbon nanotubes," Chemical Physics Letters, vol. 295, no. 4, pp. 273-278, 1998.

[11] F. Buffa, G. A. Abraham, B. P. Grady, and D. Resasco, "Effect of nanotube functionalization on the properties of single-walled carbon nanotube/polyurethane composites," Journal of Polymer Science B, vol. 45, no. 4, pp. 490-501, 2007.

[12] H. Xin, Q. Han, and X.-H. Yao, "Buckling and axially compressive properties of perfect and defective single-walled carbon nanotubes," Carbon, vol. 45, no. 13, pp. 2486-2495, 2007.

[13] Y. D. Kuang, X. Q. He, C. Y. Chen, and G. Q. Li, "Buckling of functionalized single-walled nanotubes under axial compression," Carbon, vol. 47, no. 1, pp. 279-285, 2009.

[14] Y. D. Kuang and X. Q. He, "Young's moduli of functionalized single-wall carbon nanotubes under tensile loading," Composites Science and Technology, vol. 69, no. 2, pp. 169-175, 2009.

[15] X. Q. He, Y. D. Kuang, C. Y. Chen, and G. Q. Li, "Enhanced mechanical properties of single-walled carbon nanotubes due to chemical functionalization," Journal of Physics Condensed Matter, vol. 21, no. 21, Article ID 215301, 2009.

[16] Z. Q. Zhang, B. Liu, Y. L. Chen, H. Jiang, K. C. Hwang, and Y. Huang, "Mechanical properties of functionalized carbon nanotubes," Nanotechnology, vol. 19, no. 39, Article ID 395702, 2008.

[17] B.-W. Jeong, J.-K. Lim, and S. B. Sinnott, "Elastic torsional responses of carbon nanotube systems," Journal of Applied Physics, vol. 101, no. 8, Article ID 084309, 2007.

[18] W. K. Liu, E. G. Karpov, and H. S. Park, Nano Mechanics and Materials, Science Press, Beijing, China, 2007.

[19] W. K. Liu, E. G. Karpov, S. Zhang, and H. S. Park, "An introduction to computational nanomechanics and materials," Computer Methods in Applied Mechanics and Engineering, vol. 193, no. 17-20, pp. 1529-1578, 2004.

[20] “LAMMPS Users Manual," 2009, http://lammps.sandia.gov.

[21] S. J. V. Frankland, A. Caglar, D. W. Brenner, and M. Griebel, "Molecular simulation of the influence of chemical cross-links on the shear strength of carbon nanotube-polymer interfaces," Journal of Physical Chemistry B, vol. 106, no. 12, pp. 3046-3048, 2002.

[22] S. C. Chowdhury and T. Okabe, "Computer simulation of carbon nanotube pull-out from polymer by the molecular dynamics method," Composites A, vol. 38, no. 3, pp. 747-754, 2007.

[23] V. V. Mokashi, D. Qian, and Y. Liu, "A study on the tensile response and fracture in carbon nanotube-based composites using molecular mechanics," Composites Science and Technology, vol. 67, no. 3-4, pp. 530-540, 2007. 

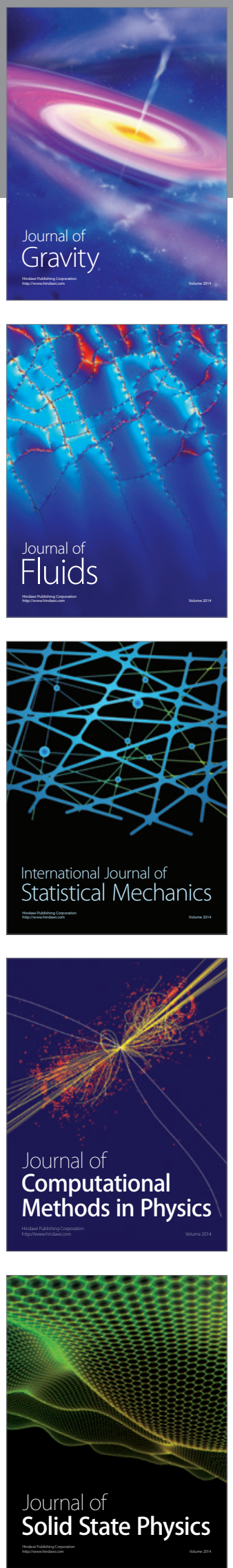

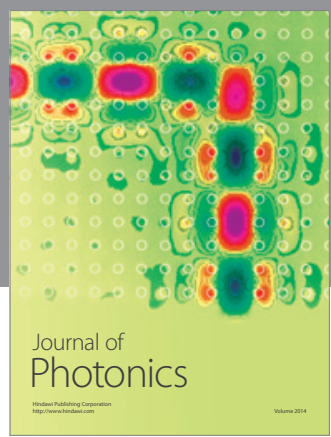

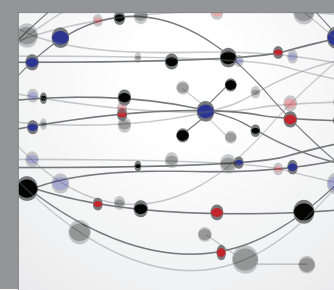

The Scientific World Journal

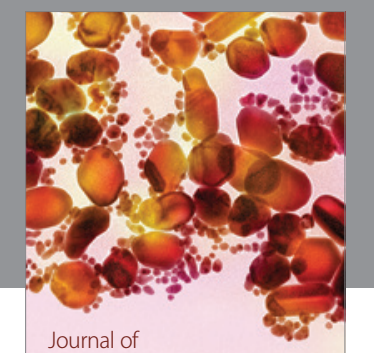

Soft Matter
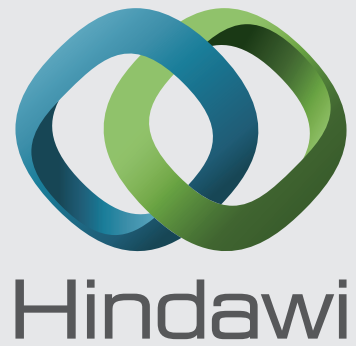

Submit your manuscripts at

http://www.hindawi.com
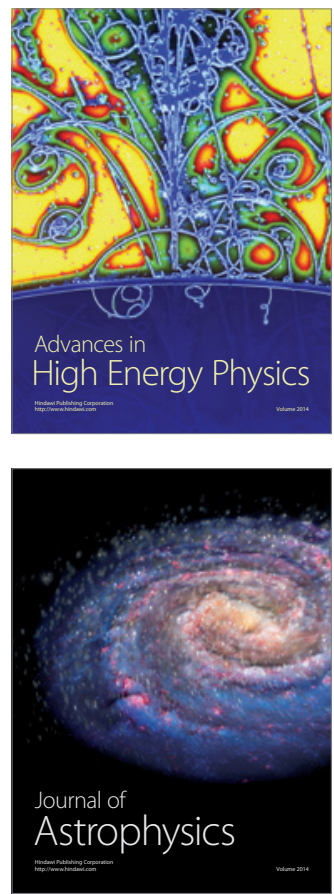
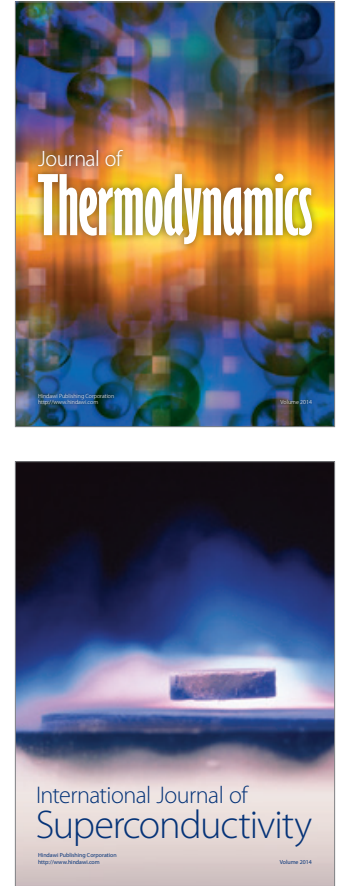
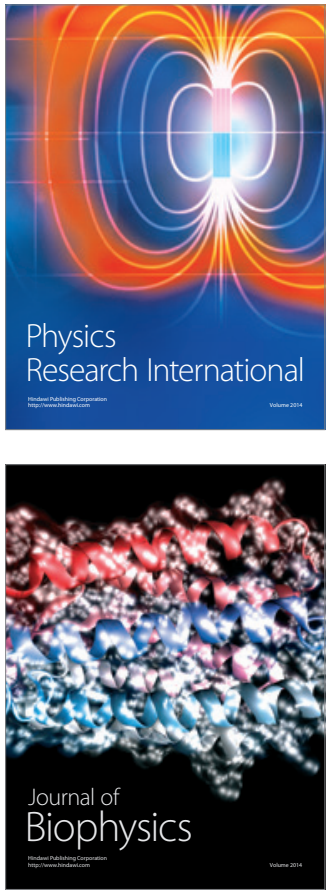
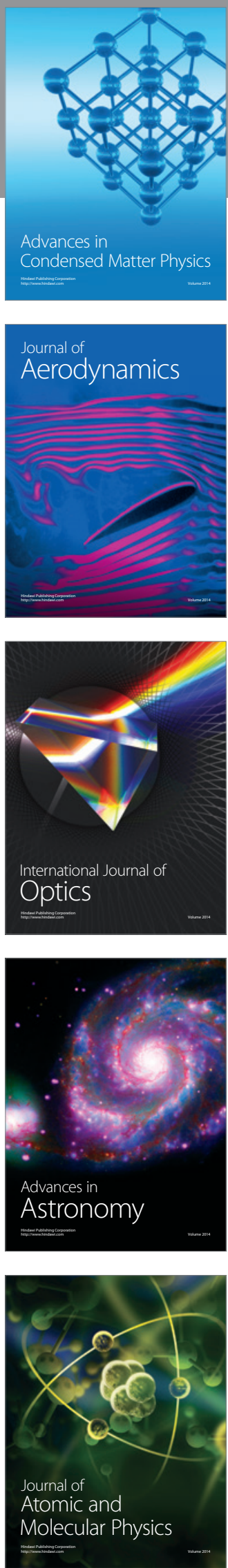